\title{
Soil: a material for bio-compatible and eco-sustainable contemporary buildings
}

\author{
D. Francese ${ }^{1}$, P. Mendonça ${ }^{2}$, L. Buoninconti ${ }^{1}$ \\ ${ }^{1}$ University of Architecture Federico II, Naples, Italy. \\ ${ }^{2}$ School of Architecture, University of Minho, Portugal.
}

For long time the research has been addressing towards the creation and the design of "high tech" components for buildings with an high performance level in any kind of situation, so as to increase a global scale dissemination and thus to ensure large market segments and wide economic incomes. This logic, based on production of goods rather than on saving resources, has provided, upon time, a number of negative effects, such as the enlargement of prime matter and pure energy source consumption, but also a massification of the architecture language and a cultural flatting, by proposing stereotypes and formal layouts which were insensitive to site diversity and peculiarity. Studies made by the authors on the Mediterranean area, its construction traditions and its social and cultural differences, focused on the presence of various invariant lines, due to similar climatic, geological, historic and human conditions, has shown that, during the whole pre-industrial period, those conditions have led to the affirmation of such techniques and materials strictly depending on the peculiar characters of each zone. The various regions, even presenting the aforesaid common and distinctive features, can be often compared to each other, and provide hints for new research currents: "low-tech" materials, and thus at low embodied energy, by exploiting a number of local resources and various configuration solutions, allowing to obtain buildings that can adapt to different site conditions, and can provide a clear saving as far as both economic and environmental items are concerned. The Rammed Earth and Adobe, even in contemporary building applications, object of the proposed paper, have been demonstrated to own various relevant characters, since they appear as good compromises between thermal mass and insulation and hold good hygroscopic regulation capacity, by absorbing and giving back the humidity to the environment. These properties allow to achieve eco-efficiency aims, by limiting resource consumption, as well as bio-compatible objectives, by providing users' health and comfort. Our research groups have carried out theoretical and experimental research that led to the chance of re-employing this traditional material within innovative products, thus considering soil, still nowadays, as a very good construction material. Case studies in Italy and Portugal are shown. 